\title{
Simulating the Effects of Intergalactic Gray Dust
}

\section{Citation}

Croft, Rupert A. C., Romeel Davé, Lars Hernquist, and Neal Katz. 2000. “Simulating the Effects of Intergalactic Gray Dust." The Astrophysical Journal 534 (2): L123-26. https:// doi.org/10.1086/312666.

\section{Permanent link}

http://nrs.harvard.edu/urn-3:HUL.InstRepos:41381802

\section{Terms of Use}

This article was downloaded from Harvard University's DASH repository, and is made available under the terms and conditions applicable to Other Posted Material, as set forth at http:// nrs.harvard.edu/urn-3:HUL.InstRepos:dash.current.terms-of-use\#LAA

\section{Share Your Story}

The Harvard community has made this article openly available.

Please share how this access benefits you. Submit a story.

\section{Accessibility}




\title{
SIMULATING THE EFFECTS OF INTERGALACTIC GREY DUST
}

\author{
Rupert A.C. Croft ${ }^{1}$, Romeel Davé ${ }^{2}$, Lars Hernquist ${ }^{1}$, And Neal Katz ${ }^{3}$
}

Draft version November 25, 2018

\begin{abstract}
Using a high-resolution cosmological hydrodynamic simulation, we present a method to constrain extinction due to intergalactic grey dust based on the observed magnitudes of distant Type IA supernovae. We apply several simple prescriptions to relate the intergalactic dust density to the gas density in the simulation, thereby obtaining dust extinctions that may be directly compared to the observed distribution of supernova magnitudes. Our analysis is sensitive to the spatial distribution of grey dust, but is not dependent on its intrinsic properties such as its opacity or grain size. We present an application of our technique to the supernova data of Perlmutter et al., who find that their high redshift sample is $\sim 0.2$ magnitudes fainter than the expectation for a non-accelerating, low-density universe. We find that for grey dust to be responsible, it must be distributed quite smoothly, e.g., tracing intergalactic gas. More realistic dust distributions, such as dust tracing the metal density, are inconsistent with observations at the $1.5-2 \sigma$ level. Upcoming observations and improved modelling of the dust distribution should lead to stronger constraints on intergalactic grey dust extinction.
\end{abstract}

Subject headings: Cosmology: observations, dust, extinction, large scale structure of Universe

\section{INTRODUCTION}

Recent observations of Type IA Supernovae (SNe) at redshifts up to $z \sim 0.8$ (Riess et al. 1998; Perlmutter et al. 1999, hereafter P99) have made possible classical cosmological tests that require standard candles, such as the magnitude-redshift relation. The most dramatic result is that these $\mathrm{SNe}$ appear dimmer (by $\sim 0.2$ magnitudes) at high redshift than would be predicted in a non-accelerating universe, suggesting at face value that we live in an accelerating universe. However, other explanations are possible, including the one we consider here, namely that distant $\mathrm{SNe}$ are dimmer due to extinction by intergalactic dust.

As distant standard candles, $\mathrm{SNe}$ are sensitive probes of extinction in the intergalactic medium (IGM). The distribution of matter in the IGM may now be modelled accurately in the context of modern cosmology using hydrodynamic simulations (see e.g., Cen et al. 1994; Hernquist et al. 1996; Davé et al. 1999). The resulting IGM is not smooth, but rather traces large-scale structure. If such structure contains not only dark matter and gas but also dust, this would result in significant variations in $\mathrm{SNe}$ brightnesses due to intervening extinction. Observationally, the distribution of Type Ia SNe magnitudes has a very small dispersion $(\widehat{\mathrm{P} 99})$. Thus by comparing simulations to the distribution of observed brightnesses, we can set limits on the amount of dust extinction and possibly constrain its spatial distribution with respect to intergalactic gas. In this Letter we present a technique for doing this, and apply it to the SNe observations of $\mathrm{P99}$.

Intergalactic grey dust has been examined in a series of papers by Aguirre (1999a,b; hereafter A99) and Aguirre \& Haiman (1999), who develop a scenario in which small grains are preferentially destroyed during ejection from galaxies, polluting the IGM with large dust grains that are effectively grey in the bandpasses of the SNe data. This greyness is necessary in order not to violate tight limits on reddening from SNe data, which imply that galactic-type dust would provide negligible absorption ( more, a significant fraction of the dust must reside in the IGM. If the grey dust causing extinction were present only in the ISM of the supernova host galaxy, this would introduce too large a dispersion in observed $\mathrm{SNe}$ magnitudes (Riess et al. 1998). In this study, we assume that grey dust blends smoothly from galaxies into the surrounding IGM. Our analysis is insensitive to the intrinsic properties of the dust, such as its opacity and grain size, since we use the simulations to directly translate the observed SNe magnitude distribution into a dust extinction in magnitudes. It is, however, sensitive to the way in which dust traces the distribution of gas in the IGM, and we will consider several simple but plausible variations of this relation.

In $\S 2$ we describe our simulations of the IGM, and of dust extinction. In $\S$ 国 we describe our analysis method and results, including constraints on grey dust afforded by current SN observations. In $\S$ 四 we discuss systematic uncertainties, and the implications of our results.

\section{SIMULATIONS OF INTERGALACTIC DUST}

We employ a hydrodynamic simulation of a $\Lambda$ dominated cold dark matter model, with $\Omega_{m}=0.4$, $\Omega_{\Lambda}=0.6, \Omega_{b}=0.02 h^{-2}, H_{0}=65 \mathrm{~km} \mathrm{~s}^{-1} \mathrm{Mpc}^{-1}$, and $\sigma_{8}=0.8$. Our simulation volume is $50 h^{-1} \mathrm{Mpc}$ with $10 h^{-1} \mathrm{kpc}$ spatial resolution, having $144^{3}$ dark matter and $144^{3}$ gas particles, and was evolved from $z=49 \rightarrow 0$ using Parallel TreeSPH (Davé, Dubinski \& Hernquist 1997).

In order to obtain dust column densities along lines of sight, we consider three different ways that dust may trace intergalactic hydrogen gas:

\footnotetext{
${ }^{1}$ Harvard-Smithsonian Center for Astrophysics, Cambridge, MA 02138; rcroft,lars@cfa.harvard.edu

${ }^{2}$ Princeton University Observatory, Princeton, NJ 08544; rad@astro.princeton.edu

${ }^{3}$ Department of Physics and Astronomy, University of Massachusetts, Amherst, MA, 01003; nsk@kaka.phast.umass.edu
} 
1. $\rho_{\text {dust }} \propto \rho_{\text {gas }}:$ Dust traces gas linearly.

2. $\rho_{\text {dust }} \propto \rho_{\text {metal }}$ : Dust traces metals linearly.

3. $\rho_{\text {dust }} \propto \rho_{\text {gas }}^{2}:$ Dust traces gas quadratically.

As our simulation makes no direct prediction for the metallicity of gas, we adopt a heuristic prescription (c.f. Cen \& Ostriker 1999), in the second case above. We assume that the metallicity is $10^{-2}$ solar if the gas overdensity is less that 10, solar if the overdensity is greater than 1000, and log-linear in between.
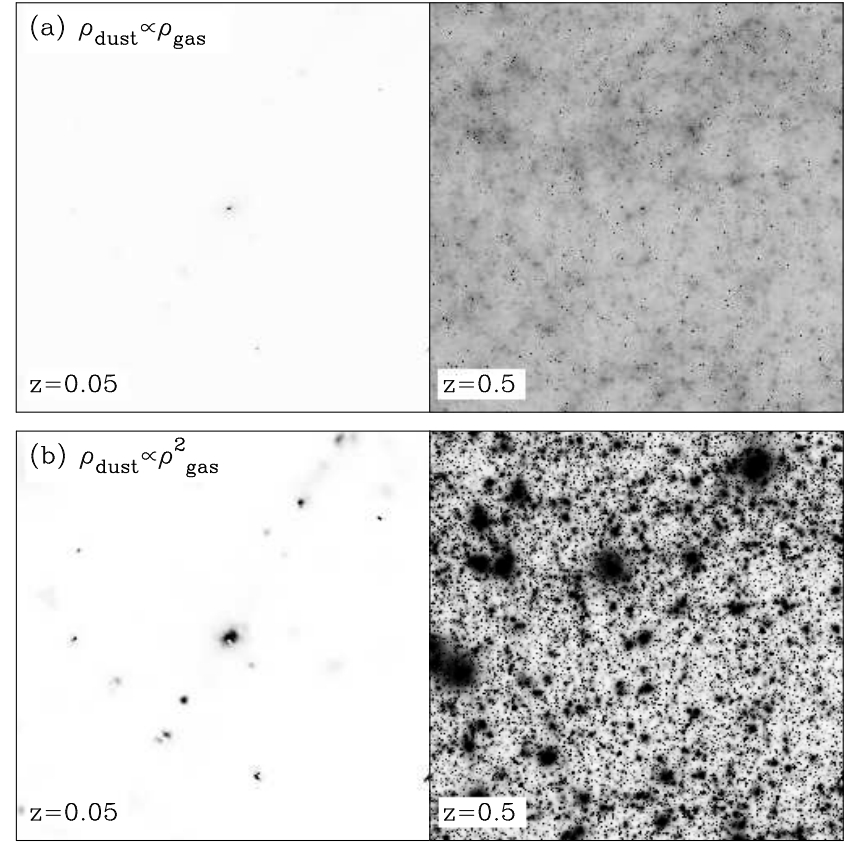

Figure 1: Dust extinction maps in $2.2^{\circ} \times 2.2^{\circ}$ patches of sky out to $z=0.05$ (left) and $z=0.5$ (right), for (a) $\rho_{\text {dust }} \propto \rho_{\text {gas }}$ and (b) $\rho_{\text {dust }} \propto \rho_{\text {gas }}^{2}$ The median extinction magnitude to $z=0.5$ is set equal to 0.4 for both (a) and (b). The maps correspond to what would be seen against a white background.

To extract dust extinction values from the simulations, we assume that the gas associated with each particle is spread over its SPH smoothing volume (see e.g., Hernquist \& Katz 1989). We perform a numerical integration of gas column density along 5000 rays cast through these volumes, at the same time applying one of the three transformations given above to relate gas to dust densities. To reach the required path lengths out to $z \sim 0.5$, we follow rays through 26 simulation volumes, each ray entering through a random point on a random face. This yields the column density of dust to $z=0.5$ along each line of sight.

In Figure 1 we show extinction maps of $2.2^{\circ} \times 2.2^{\circ}$ patches of sky, for (a) $\rho_{\text {dust }} \propto \rho_{\text {gas }}$ and (b) $\rho_{\text {dust }} \propto \rho_{\text {gas }}^{2}$. The median extinction to $z=0.5$ was set to be equal (to $0.4 \mathrm{mag}$ ) for cases (a) and (b). In Figure 2, we show how the mean dust extinction and its dispersion varies with redshift. Here, the dispersion in all three panels was set to be the same small value, equal to the difference in quadrature of the dispersion in $\mathrm{SNe}$ magnitudes at high $\left(\sigma_{z=0.5}=0.157\right)$ and low $\left(\sigma_{z=0.05}=0.154\right)$ redshifts observed by P99, namely 0.03 mag. Figs. 1 and 2 show that the mean extinction is much greater when the dust is more smoothly distributed. We will now quantitatively explore the constraints that can be put on the dust extinction by using the observed distribution of SNe magnitudes.

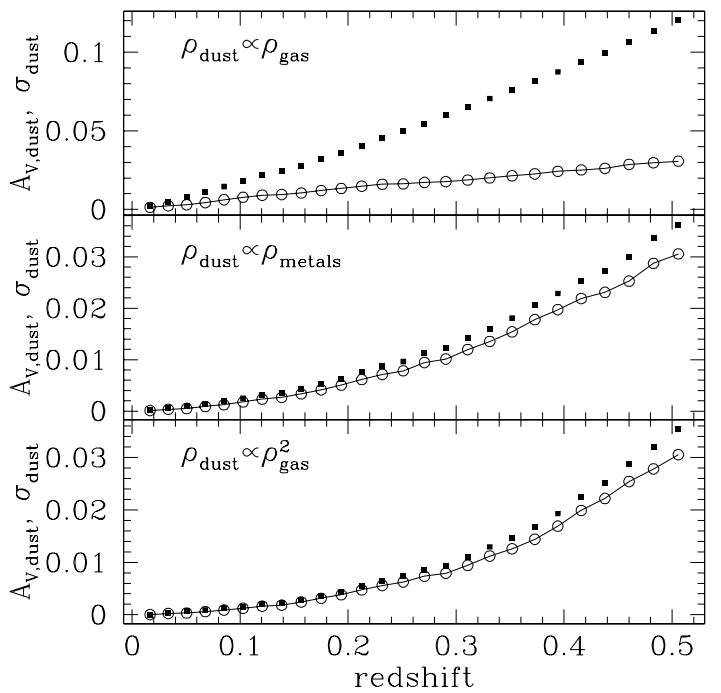

Figure 2: Mean and dispersion of the dust extinction as a function of redshift. Filled squares show mean, open circles with line show the dispersion. Values have been scaled to a dispersion of 0.03 magnitudes at $z=0.5$.

\section{COMPARISON WITH OBSERVATIONS}

We make use of two characteristics of the observed SN data in our comparison, the change with redshift of the dispersion in SNe magnitudes, and the shape of the histogram of SNe magnitudes. As mentioned above, P99 found little difference in the dispersions of two samples with $\bar{z} \sim 0.05$ and $\bar{z} \sim 0.5$. As they stated, this leaves little room for dispersion due to dust, as this dispersion is expected to increase for longer path lengths (see Fig. 2). In order to quantify this, and the effect of the distribution shape, we generate simulated SNe magnitudes, and compare them to the P99 data using a maximum likelihood approach.

The observational datasets we use are both taken from P99 (their Tables 1 and 2), being the high- $z$ SNe of the Supernova Cosmology Project, and the low- $z$ sample of Calán-Tololo SNe survey (Hamuy et al. 1996). We use 40 (non-reddened SNe) of the former SNe, between $z=0.172$ and $z=0.83(\bar{z} \sim 0.5)$, and 16 of the latter SNe which lie between $z=0.02$ and $z=0.101(\bar{z} \sim 0.05)$.

We generate simulated datasets for each dust model described in $\S 2$. For each dust model, we vary two parameters; first, $M_{C}$, a cosmological magnitude shift applied to all simulated SNe at a given $z$, normalized so that $M_{C}=0$ at $z=0.5$ corresponds to the best fitting cosmology found by P99 (with $\Omega_{m}=0.28$ and $\Omega_{\Lambda}=0.72$ ); and second, $A_{V}$, the median $\mathrm{V}$-band magnitude of dust extinction out to $z=0.5^{1} . M_{C} \approx-0.2$ then corresponds to an open model with $\Omega_{m} \sim 0.3$, and $M_{C} \approx-0.4$ to an $\Omega_{m}=1$ model. We generate the simulated datasets as follows:

(1) We renormalize the 5000 simulated lines-of-sight so that the median dust extinction to $z=0.5$ equals $A_{V}$.

(2) We add the cosmological shift, $M_{C}$.

(3) We broaden the magnitude distribution, which involves convolving it separately with a Gaussian of width given by the observational error of each $\mathrm{SN}$, and then adding these

\footnotetext{
${ }^{1}$ We use the median in order to be less sensitive to long tails of the distribution (c.f. Figure 3) when renormalizing extinction values.
} 
distributions together. When doing this, we include an "intrinsic" SN dispersion of $\sigma_{\text {int }}=0.17$ mag ( $($ P99 $)$. Varying this (by $\pm 0.1 \mathrm{mag}$ ) makes little difference to the results (see $\S 4$ ).

(4) We truncate the distribution at a magnitude difference $\Delta_{M}=1$, to roughly account for the fact that SNe along lines of sight passing through high extinction regions would not make it into these magnitude-limited samples.

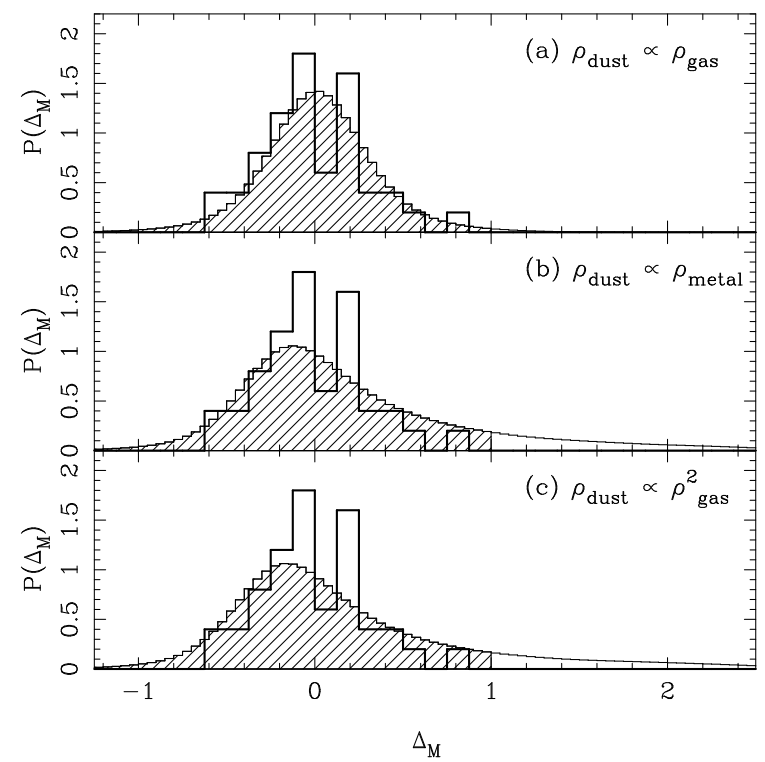

Figure 3: The PDF of magnitude differences, $\Delta_{M}$, for 5000 simulated SNe. The shaded region is included in the calculation of the dispersion; non-shaded region indicates heavily obscured SNe that are not included in the PDF normalization. The histogram shows 40 of the high- $z$ SNe of $\mathrm{P} 99$.

We derive the probability distribution function (PDF) of SNe magnitude differences $\Delta_{M}$ predicted by the simulation, so that the predicted number of SNe between $\Delta_{M}$ and $\Delta_{M}+d \Delta_{M}$ is $N P\left(\Delta_{M}\right) d \Delta_{M}$, where $N$ is the number of observed SNe. Figure 3 shows histograms of the PDF of $\Delta_{M}$, for $A_{V}=0.4 \mathrm{mag}$ and $M_{C}=-0.4$. We also plot the observational data of P99. In effect, for this plot, we have brightened the simulated SNe to mimic an $\Omega_{m}=1$ model and then dimmed them with dust. We can see that in panel (a), where the dust is fairly smoothly distributed, the simulated PDF is not too different from the observations. In the other panels, which have clumpier dust, there is a skewness not seen in the observed data, due to a long tail of high extinction lines-of-sight.

For each set of simulated lines of sight, we form the relative likelihood of drawing all the observed SNe magnitudes, $\mathcal{L}=\prod_{i}^{N} P\left(\Delta_{M, i}\right)$, where $\Delta_{M, i}$ is the magnitude difference of SN $i$. We define the quantity $S=-2 \ln \mathcal{L}$ and assume that $S$ follows a $\chi^{2}$ distribution in order to derive confidence limits on the parameters $M_{C}$ and $A_{v}$. In the present analysis, we use results at two different redshifts ( $z=0.05$ and $z=0.5$ ), and combine the two by adding the values of $S$. It is this step, combining the likelihoods at two different redshifts, which constrains the amount of additional dispersion (or the change in the shape of the magnitude distribution) due to grey dust between low and high redshifts. Contours of $\Delta S=2.3,6.2$, and 11.8 (the difference in $S$ from its minimum value), representing 1,2 and $3 \sigma$ intervals of joint confidence, are plotted in Figure 4.

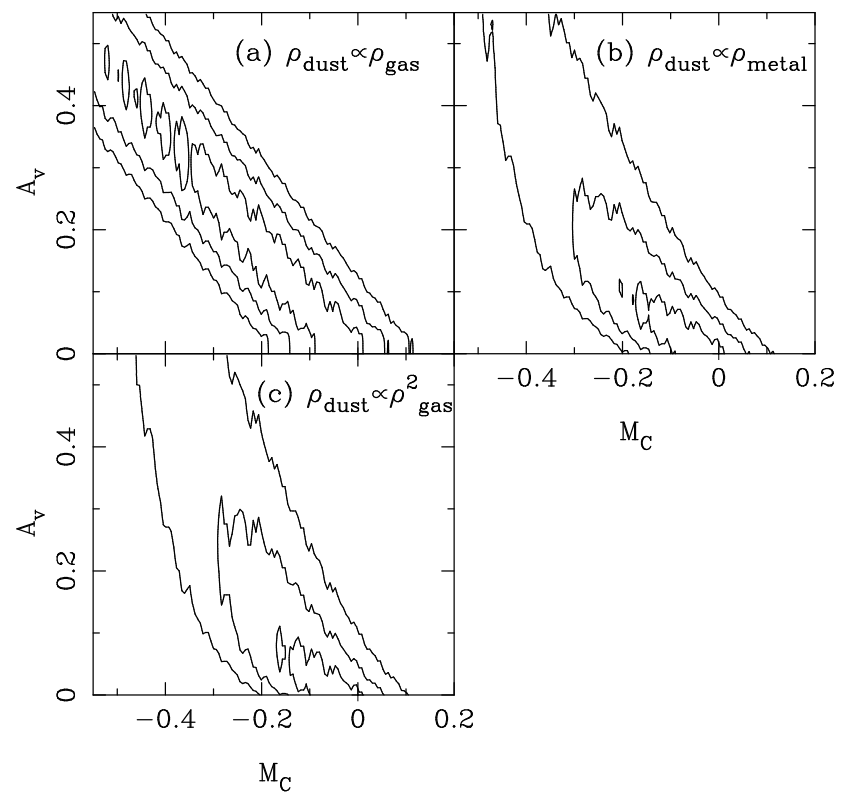

Figure 4: 1, 2 and $3 \sigma$ contours for the cosmological magnitude shift, $M_{C}$, with respect to a model with $\Omega_{\Lambda}=0.72$ and $\Omega_{M}=0.28$, and the median extinction due to grey dust $\left(A_{V}\right)$.

Figure 4 shows that the smoothest distribution of dust easily accomodates enough extinction to reconcile a nonaccelerating $\left(M_{C}=-0.2\right)$ or flat $\left(M_{C}=-0.4\right)$ universe, as there is a strong degeneracy between the cosmological magnitude shift and dust extinction. The other cases, where dust traces metals and $\rho_{\text {gas }}^{2}$, are coincidentally quite similar. Such a distribution of dust would be mildly disfavored in a non-accelerating open universe and ruled out at $\sim 99 \%$ confidence in an Einstein-de-Sitter universe. The constraints arise mostly due to the shape of the distribution; the fact that the observed dispersions are similar at $z=0.05$ and $z=0.5$ is relatively unimportant, only making a noticeable difference in panel (a) where the shape is similar to the observed distribution.

\section{DISCUSSION}

We have found that current $\mathrm{SNe}$ datasets appear to have some power to constrain grey dust models. We find it somewhat unlikely (at the $1.5-2$ level) that sufficient grey dust could be distributed in the relatively clumpy fashion expected for the metal distribution (c.f., Gnedin \& Ostriker 1997, Davé et al. 1998, Cen \& Ostriker 1999) to reconcile the P99 data with a non-accelerating universe. This suggests that any substantial grey dust component must be largely segregated from the galaxies where it was formed. In the specific grey dust model of A99, sputtering does destroy dust more effectively in denser regions, but once in the IGM the large grains have long lifetimes, so that dust is still likely to trace the metals. The question of how the dust and metals are distributed can be answered self-consistently by modelling the relevant physical processes (dust and metal ejection from galaxies) directly in the simulations (e.g., Aguirre et al., in preparation).

There are many alternative explanations for SNe appearing dimmer in the past. For example, there may be metallicity effects in the host galaxy (Höflich et al. 1999), intrinsic evolution (Riess et al. 1999), observational selection differences between CCDs used for distant $\mathrm{SNe}$ 
and photographic plates used for nearby samples Howell, Wang \& Wheeler 1999), or time evolution of the gravitational constant (Amendola, Corasaniti \& Occhionero 1999; Garcia-Berro et al. 1999). Such effects would alter the interpretation of our parameter $M_{C}$. Gravitational lensing magnification (Metcalf 1999) would also change the magnitude dispersion at higher redshifts.

The study we have presented is reasonably general, in that our analysis is not dependent on the (unknown) microscopic properties of this hypothetical intergalactic dust. Still, there are some possible systematic uncertainties, which we now consider. We assume (as do P99) that the observed dispersion in $\mathrm{SNe}$ magnitudes in excess of the estimated observational errors is an intrinsic property of $\mathrm{SNe}$ and does not vary with redshift. One could envision scenarios in which the intrinsic dispersion is lower at high redshift, thus allowing more dispersion from dust. However, as mentioned earlier, most of the statistical power of our analysis comes from the shape of the distributions, which is not significantly affected by a change in the intrinsic dispersion. Also, we find that when we decrease the intrinsic SN dispersion $\left(\sigma_{\text {int }}\right)$, models with more dust fit slightly better at high- $z$, but the low- $z$ fit becomes worse, a trade-off which means that the overall results hardly change. The distribution shape depends on our assumption that the intrinsic dispersion has a Gaussian distribution in magnitudes, whereas a distribution skewed to fainter magnitudes could weaken our constraints. With a larger sample of low-z SNe we might be in a position to test this, by using the distribution of low-z SN magnitudes to make a simulated high-z sample with the correct intrinsic distribution shape. Also, we have made a simplifying approximation in our simulated high-z samples, by using dust extinction magnitudes that result from integrating the dust contribution from $z=0$ to exactly $z=0.5$. The real $\mathrm{SNe}$ are at a range of redshifts; our constraints are effectively conservative because of this. We have also assumed that the total mass of dust does not change with redshift from $z=0.5$ to $z=0$; dust increasing with time would strengthen our constraints, while a decrease seems implausible. Finally, the truncation of our simulated magnitude distribution at $+1 \mathrm{mag}$ is a rather approximate procedure. We have tried changing the cutoff, and find that with none (an unrealistic case) the constraints become stronger, as we might expect. With a lower cutoff, the right side of the observed distribution is not reproduced. One additional degree of freedom would involve changing the functional form of the cutoff. In the future, as observations improve, we plan to simulate such observational selection effects more carefully.

On the simulation side, our relatively low mass resolution $\left(m_{\text {gas }}=8.5 \times 10^{8} M_{\odot}\right)$ means that we miss fluctuations in the extinction that occur on smaller mass scales. If we had higher resolution, this should have the effect of making our dust constraints tighter. We find that the dispersion in projected magnitudes largely depends on small scale fluctuations. We tested this by splitting the simulation into small sub-volumes and then shuffling them to remove large scale correlations (see e.g., Bhavsar \& Ling 1988) before projection, and we obtained similar results. Changing the assumed cosmological model could have some effects, although it is difficult to conceive of models which have less power on small scales while fitting other constraints (see e.g., White \& Croft 2000).

The models of A99 have grains that do produce some reddening, typically in the infrared. A complementary approach to ours is therefore to use information from different color bands. Such an approach was used by P99, for example, to constrain normal galactic-type dust. Recent near-IR observations by Riess et al. (2000) have made reddening constraints tight even for non-standard large dust grains. Our approach does not make any use of color information, and so constrains the most extreme scenario in which the dust is totally "grey".

In summary, we have used cosmological hydrodynamic simulations to explore how intergalactic grey dust could affect observations of high redshift supernovae, and how supernova data can constrain grey dust extinction. We conclude that only a fairly smooth distribution of dust could readily mimic the effect of an accelerating Universe. Such a distribution would be strange, as the dust would be strongly biased away from metal producing regions. More realistic dust distributions are mildly disfavored, but upcoming samples of SNe (at current redshifts) should enable us to put tighter limits as they more precisely determining the shape of the brightness distribution. At higher redshifts $(z \gtrsim 1)$, grey dust predicts that $\mathrm{SNe}$ should show increased dimming, while decreased dimming would be strong evidence for a cosmological constant.

We thank Anthony Aguirre, Bruce Draine, and Bob Kirshner for useful discussions, and David Weinberg for helpful comments on the manuscript.

\section{REFERENCES}

Aguirre, A., 1999a, ApJ, 512, L19 [A99]

Aguirre, A., 1999b, ApJ, in press

Aguirre, A., \& Haiman, Z., 1999, ApJ, submitted, astro-ph/9907030

Amendela, I., Corasaniti, S. \& Occhionero, F. Ig99, astro$\mathrm{ph} / 9907222$

Bhavsar, S. \& Ling, E. N. 1988, ApJ, 331, L63

Cen, R., Miralda-Escudé, J., Ostriker, J. P. \& Rauch, M. 1994, ApJ, 427, I. 9

Cen, R. \& Ostriker, J. P. 1999, ApJ, 519, L109

Davé, R., Hellsten, U., Hernquist, L., Katz, N. \& Weinberg, D. H. 1998, ApJ, 509, 661

Davé, R., Dubinski, J., \& Hernquist, L. 1997, NewAst, 2, 71

Davé, R., Hernquist, L., Katz, N. \& Weinberg, D. H. 1999, ApJ, 511, 521

Garcia-Berro, E., E., Isern, J., Benvenuto, O., Althaus, L. 1999 tro $/ 9007440$

Gnedin, N.Y. \& Ostriker, J. P. 1997, ApJ, 486, 581
Hamuy, M., Phillips, M.M. Maza, J., Suntzeff, N.B., Schommer, R.A. \& Aviles, R., 1996, ÄJ, 112, 2391

Hernquist, L. \& Katz, N. 1989, ApJS, 70, 419

Hernquist, L., Katz, N., Weinberg, D. H. \& Miralda-Escudé, J. 1996, ApJ, 457, L51

Höflich, P, Nomata,K, Imeda, H. \& Wheeler, J. C. 1999, ApJ, in press, astro-ph/9908226

Howell, A Wang, L. \& Wheeler, J. C. 1999, ApJ, in press, astro$\mathrm{ph} / 9908127$

Metcalf, R.B., 1999, MNRAS, 305, 746

Perlmutter, S., et al., 1999, ApJ, 517, 565 [P99]

Riess, A. G., Fillipenko A V, Li, W. \& Schmidt, B. 1999, ApJ, submitted, astro-ph/9907038

Riess, A.G., et al., 1998, AJ, 116, 1009

Riess, A.G., et al., 2000, ApJ, in press, astro-ph/0001384

White, M. \& Croft, R. A. C., 2000, ApJ, submitted, astro$\mathrm{ph} / 0001247$ 